\title{
Repeated Mechanical Thrombectomy in Recurrent Large Vessel Occlusion Acute Ischemic Stroke
}

\author{
Mehdi Bouslama ${ }^{a}$ Diogo C. Haussen ${ }^{a}$ Leticia C. Rebello ${ }^{a}$ \\ Jonathan A. Grossberg ${ }^{b}$ Michael R. Frankel ${ }^{a}$ Raul G. Nogueira ${ }^{a}$ \\ Departments of a Neurology and ${ }^{b}$ Neurosurgery, Grady Memorial Hospital and Emory \\ University School of Medicine, Atlanta, Ga., USA
}

\section{Key Words}

Acute stroke $\cdot$ Cerebral ischemia $\cdot$ Recurrent event $\cdot$ Thrombectomy $\cdot$ Outcomes

\begin{abstract}
Background: Endovascular therapy has been proven effective for the treatment of large vessel occlusion strokes (LVOS). However, the feasibility and potential benefits of repeat thrombectomy for recurrent stroke is unclear. We aim to report our experience with repeat thrombectomy for recurrent LVOS. Methods: We reviewed our prospectively collected endovascular database for patients who underwent repeated mechanical thrombectomy. Baseline characteristics, procedural data and outcomes were evaluated. Patients with repeat thrombectomy were compared to patients with single thrombectomy. For patients with repeat thrombectomy, imaging and procedural variables were compared between first and last procedures. $\boldsymbol{R e}$ sults: Out of 697 patients treated within the study period, 15 patients (2\%) had repeat thrombectomies (14 treated twice and one thrice). The mean age was $63 \pm 15$ years and $40 \%$ were males. The median time between the first and last procedure was 18 (1-278) days. Cardioembolism (66\%) was the most common etiology, followed by intracranial atherosclerosis (13\%) and large vessel atherosclerosis (6\%). At 90 days after the last thrombectomy, $60 \%$ of patients achieved a modified Rankin Scale score of $0-2$ and $20 \%$ were deceased. There were no statistically significant differences in demographics, stroke severity, time from last known normal to puncture, reperfusion rates, hemorrhagic complications, good clinical outcomes and mortality between patients who underwent repeat thrombectomy and those who had a single thrombectomy. Conclusion: In properly selected patients suffering recurrent LVOS, repeated mechanical thrombectomy appears to be feasible and safe. A previous thrombectomy should not discourage aggressive treatment as these patients may achieve similar rates of good clinical outcomes as those who undergo single thrombectomy.


Bouslama et al.: Repeated Mechanical Thrombectomy in Recurrent Large Vessel Occlusion Acute Ischemic Stroke

\section{Introduction}

Endovascular therapy has been proven effective for the treatment of large vessel occlusion stroke (LVOS) and has become the standard of care for patients presenting early in the therapeutic window [1]. Nearly $25 \%$ of stroke victims will have a recurrent event within 5 years [2]. However, the specific rate of LVOS recurrence is unknown.

Although repeated thrombolytic therapy has been reported safe and beneficial for recurrent strokes [3-6], the data regarding repeat thrombectomy (RT) is scarce and limited to a few case reports [7-9]; therefore, its safety and potential efficacy remain elusive. We aim to report our local experience with RT for recurrent LVOS. Our specific objectives are to (1) demonstrate the frequency of RT cases, (2) compare the clinical and procedural characteristics of patients that undergo RT and those who have a single thrombectomy, and (3) evaluate the safety and efficacy of RT.

\section{Methods}

This was a retrospective review of a prospectively collected database for RT cases between June 2012 and March 2016. Imaging and procedural variables were compared between first and last procedures. The RT cases were then compared to consecutive patients treated with single thrombectomy across a similar period. Successful reperfusion was defined as per modified Thrombolysis in Cerebral Infarction (mTICI) score 2b-3 [10]. Hemorrhagic complications were defined as per European Cooperative Acute Stroke Study (ECASS) criteria [11]. Good outcome was defined as per modified Rankin Scale (mRS) 0-2 at 90 days [12]. Alberta Stroke Program Early CT score (ASPECTS) was used to quantify infarct extent at baseline [13]. This study was approved by the local institutional review board.

\section{Statistical Analysis}

Continuous variables were reported as mean \pm SD or median (IQR), as appropriate. Categorical variables were reported as proportions. Between groups, comparisons for continuous/ordinal variables were made with Student's t test, Mann-Whitney U test, paired t test, or ANOVA, as appropriate. Categorical variables were compared by $\chi^{2}$ test or Fisher's exact test as appropriate. Significance is set at $p<0.05$. Statistical analysis was performed using IBM ${ }^{\circledR}$ SPSS $^{\circledR}$ Statistics 23 (IBM ${ }^{\circledR}$, Armonk, N.Y., USA).

\section{Results}

Fifteen (2\%) of 697 patients who underwent endovascular treatment for acute ischemic stroke (AIS) over the study period were treated with RT. Fourteen patients were treated twice and one thrice (table 1; fig. 1). The mean age at the time point of first thrombectomy was 62.7 years. The median time interval between both endovascular treatments was 18 (1-278) days. The mean last known normal to puncture time interval was $245 \pm 99$ min for the first procedure and $486 \pm 1,131 \mathrm{~min}$ for the last procedure $(\mathrm{p}=0.44)$. The mean baseline National Institute of Health Stroke Scale (NIHSS) was $15.9 \pm 6.1$ for the first and $21.2 \pm 7.2$ for the last thrombectomy ( $p=0.03)$. The mean ASPECTS was $8 \pm 1.65$ for the first and $8.36 \pm$ $1.55(p=0.41)$ for the last procedure. Hospitalized patients $(n=5)$ had nonsignificant shorter time from last known normal to puncture in the last procedure as compared to nonhospitalized patients ( $\mathrm{n}=10,111 \pm 25$ vs. $801 \pm 1,340 \mathrm{~min}, \mathrm{p}=0.14$ ), but outcomes were comparable. Successful reperfusion was achieved in 14/15 patients after the first thrombectomy and in all patients after RT. No vascular complications were identified. No parenchymal hemorrhage was observed after the first procedure and two were noted after the repeated intervention. The rate of good clinical outcome at 90 days in RT patients was $60 \%$ and their 90 -day mortality rate was only $20 \%$. Stroke etiology was the same for first and recurrent 
Bouslama et al.: Repeated Mechanical Thrombectomy in Recurrent Large Vessel

Table 1. Demographic, clinical, neuroradiological and procedural parameters as well as outcome measures before and after each procedure

\begin{tabular}{|c|c|c|c|c|c|c|c|c|c|c|c|c|c|}
\hline \multirow[t]{2}{*}{ Case } & \multirow{2}{*}{$\begin{array}{l}\mathrm{Age}^{\mathrm{a}} / \\
\mathrm{sex}\end{array}$} & \multirow{2}{*}{$\begin{array}{l}\text { Stroke } \\
\text { etiology }\end{array}$} & \multirow{2}{*}{$\begin{array}{l}\text { Time between } \\
\text { procedures, } \\
\text { days }\end{array}$} & \multicolumn{10}{|c|}{ Procedure characteristics } \\
\hline & & & & $\begin{array}{l}\text { proce- } \\
\text { dure }\end{array}$ & $\begin{array}{l}\text { baseline } \\
\text { mRS }\end{array}$ & NIHSS & ASPECTS & site (side) & $\begin{array}{l}\text { LKN to } \\
\text { puncture, } \\
\text { min }\end{array}$ & device & mTICI & bleed & $\begin{array}{l}\text { 90-day } \\
\text { mRS }\end{array}$ \\
\hline \multirow[t]{2}{*}{1} & \multirow[t]{2}{*}{$55 / \mathrm{M}$} & \multirow[t]{2}{*}{ APS } & \multirow[t]{2}{*}{26} & 1 st & 0 & 18 & 9 & M1 (L) & 103 & $\mathrm{TA}+\mathrm{SR}$ & $2 \mathrm{~b}$ & none & 2 \\
\hline & & & & 2nd & 2 & 13 & 10 & M1 (L) & 113 & TA & $2 b$ & none & 1 \\
\hline \multirow[t]{2}{*}{2} & \multirow[t]{2}{*}{$82 / \mathrm{F}$} & \multirow[t]{2}{*}{ CE } & \multirow[t]{2}{*}{12} & $1 \mathrm{st}$ & 0 & 20 & 9 & M1 (R) & 284 & $\mathrm{TA}+\mathrm{SR}$ & $2 b$ & none & 5 \\
\hline & & & & 2nd & 0 & 16 & 9 & M1 (L) & 130 & SR & 3 & none & 6 \\
\hline \multirow[t]{2}{*}{3} & \multirow[t]{2}{*}{$60 / \mathrm{M}$} & \multirow[t]{2}{*}{ ICAD } & \multirow[t]{2}{*}{18} & 1 st & 1 & 14 & 10 & VB & 257 & ICS & 3 & none & 2 \\
\hline & & & & 2nd & 2 & 15 & 10 & VB & 133 & TA+ICS & $2 \mathrm{~b}$ & none & 2 \\
\hline \multirow[t]{2}{*}{4} & \multirow[t]{2}{*}{$52 / \mathrm{F}$} & \multirow[t]{2}{*}{$\mathrm{CE}$} & \multirow[t]{2}{*}{278} & $1 \mathrm{st}$ & 0 & 28 & 8 & M1 (L) & 203 & TA & 3 & none & 1 \\
\hline & & & & 2nd & 0 & 20 & 9 & M1 (R) & 596 & $\mathrm{TA}+\mathrm{SR}$ & 3 & $\mathrm{PH}-2$ & 4 \\
\hline \multirow[t]{3}{*}{5} & \multirow[t]{3}{*}{$32 / \mathrm{F}$} & \multirow[t]{3}{*}{$\mathrm{CE}$} & \multirow[t]{2}{*}{616} & $1 \mathrm{st}$ & 0 & 9 & 9 & M1 (L) & 270 & TA & $2 \mathrm{~b}$ & none & 1 \\
\hline & & & & 2nd & 3 & 12 & 8 & $\mathrm{M} 1+\mathrm{M} 2(\mathrm{R})$ & 240 & TA & $2 b$ & none & 1 \\
\hline & & & 45 & 3rd & 0 & 12 & 8 & M1 (L) & 202 & $\mathrm{TA}+\mathrm{SR}$ & $2 \mathrm{~b}$ & none & 1 \\
\hline \multirow[t]{2}{*}{6} & $61 / \mathrm{M}$ & ICAD & 1 & $1 \mathrm{st}$ & 0 & 12 & 10 & M1 (R) & 475 & $\mathrm{TA}$ & $2 \mathrm{~b}$ & none & 2 \\
\hline & & & & $2 n d^{b}$ & 0 & 13 & - & $\mathrm{M} 1+\mathrm{M} 2(\mathrm{R})$ & - & $\mathrm{SR}+\mathrm{ICS}$ & $2 \mathrm{~b}$ & none & 2 \\
\hline 7 & $57 / F$ & $\mathrm{CE}$ & 15 & $1 \mathrm{st}$ & 2 & 18 & 8 & M3 (R) & 269 & $\mathrm{TA}$ & $2 a$ & none & 3 \\
\hline & & & & 2nd & 2 & 33 & 6 & M1 (L) & 250 & SR & $2 \mathrm{c}$ & $\mathrm{a}-\mathrm{SAH}$ & 3 \\
\hline 8 & $78 / \mathrm{F}$ & $\mathrm{CE}$ & 915 & $1 \mathrm{st}$ & 0 & 13 & 8 & M1 (R) & 215 & SR & 3 & none & 1 \\
\hline & & & & 2nd & 1 & 19 & 10 & ICA-T (L) & 1,628 & SR & $2 b$ & none & 2 \\
\hline 9 & $84 / F$ & $\mathrm{CE}$ & 1 & 1 st & 2 & 13 & 6 & ICA-T (R) & 204 & SR & 3 & none & 6 \\
\hline & & & & 2nd & 2 & 21 & 10 & E-ICA+ICA-T (L) & 90 & $\mathrm{TA}+\mathrm{SR}$ & 3 & none & 6 \\
\hline 10 & $61 / \mathrm{F}$ & $\mathrm{CE}$ & 32 & $1 \mathrm{st}$ & 1 & 23 & 5 & M1 (L) & 225 & TA & $2 c$ & $\mathrm{Hi}-2$ & 1 \\
\hline & & & & 2nd & 3 & 31 & 8 & M1 (R) & 218 & $\mathrm{TA}+\mathrm{SR}$ & $2 c$ & none & 1 \\
\hline$\overline{11}$ & $63 / \mathrm{F}$ & LVA & $<1$ & $1 \mathrm{st}$ & 1 & 18 & 9 & $\mathrm{E}-\mathrm{ICA}+\mathrm{M} 1$ (L) & 573 & SR & $2 b$ & none & 2 \\
\hline & & & & 2nd & 1 & 19 & 5 & $\mathrm{E}-\mathrm{ICA}+\mathrm{M} 2(\mathrm{~L})$ & 125 & $\mathrm{SR}+\mathrm{ECS}$ & $2 b$ & PH-2 & 2 \\
\hline 12 & $62 / \mathrm{M}$ & $\mathrm{CE}$ & 876 & $1 s t$ & 1 & 21 & 6 & M1 (L) & 264 & SR & 3 & $\mathrm{Hi}-2$ & 4 \\
\hline & & & & 2nd & 4 & 35 & 9 & M1 (R) & 4,389 & SR & 3 & $\mathrm{Hi}-1$ & 6 \\
\hline 13 & $51 / \mathrm{F}$ & FMD & $<1$ & $1 \mathrm{st}$ & 0 & 16 & 6 & ICA-T+M1 (R) & 125 & $\mathrm{TA}+\mathrm{SR}$ & 3 & none & $1^{\mathrm{c}}$ \\
\hline & & & & 2nd & 0 & 17 & 7 & $\mathrm{ICA}-\mathrm{T}+\mathrm{M} 2+\mathrm{M} 3(\mathrm{R})$ & 90 & SR & $2 \mathrm{~b}$ & none & $1^{\mathrm{c}}$ \\
\hline 14 & $54 / \mathrm{M}$ & $\mathrm{CE}$ & 3 & $1 \mathrm{st}$ & 3 & 14 & 7 & ICA-T (L) & 335 & SR & 3 & none & $2^{\mathrm{d}}$ \\
\hline & & & & 2nd & 3 & 25 & 8 & $\mathrm{M} 1(\mathrm{R})$ & 140 & SR & 3 & none & $2^{\mathrm{d}}$ \\
\hline 15 & $89 / \mathrm{M}$ & $\mathrm{CE}$ & 214 & $1 \mathrm{st}$ & 0 & 2 & 9 & M1 (R) & 166 & TA & $2 b$ & none & 1 \\
\hline & & & & 2nd & 1 & 21 & 8 & ICA-T (R) & 316 & SR & 3 & none & $5^{\mathrm{e}}$ \\
\hline
\end{tabular}

APS = Antiphospholipid syndrome; a-SAH = asymptomatic subarachnoid hemorrhage; $\mathrm{CE}=$ cardioembolic; ECS = extracranial stenting; E-ICA = extracranial internal carotid artery; F = female; FMD = fibromuscular dysplasia; Hi = hemorrhagic infarction; ICAD = intracranial atherosclerotic disease; ICA-T = internal carotid artery terminus; ICS = intracranial stenting; $\mathrm{L}=$ left; LKN $=$ last known normal; LVA = large vessel atherosclerosis; $\mathrm{M}=$ male; $\mathrm{PH}=$ parenchymal hemorrhage; $\mathrm{R}=$ right; $\mathrm{SR}=$ stentriever; $\mathrm{TA}=$ thromboaspiration; VB $=$ vertebrobasilar. ${ }^{a}$ Age at first procedure. ${ }^{b}$ The patient remained symptomatic with an NIHSS of $12-13$ and was taken back to the angiography suite for further clot retrieval. ${ }^{\mathrm{c}} \mathrm{mRS}$ at 2 months. ${ }^{\mathrm{d}} \mathrm{mRS}$ at discharge. ${ }^{\mathrm{e}}$ Patient had a third recurrent stroke (not amenable to endovascular treatment) 2 weeks after discharge and was transferred to hospice.

strokes, and was categorized as cardioembolic in $66 \%$ of the patients, followed by intracranial atherosclerotic disease (13\%), and extracranial atherosclerosis (6\%).

Patients with RT were comparable to those who had a single thrombectomy in terms of clinical, procedural and outcome parameters except for higher rates of hypertension $(\mathrm{p}=$ 0.01 ) and anticoagulation ( $\mathrm{p}=0.001)$ (table 2).

\section{Discussion}

We report the largest series of patients undergoing repeat mechanical thrombectomy for recurrent LVOS, and demonstrate that RT is feasible and appears to be safe and effective in properly selected cases. 

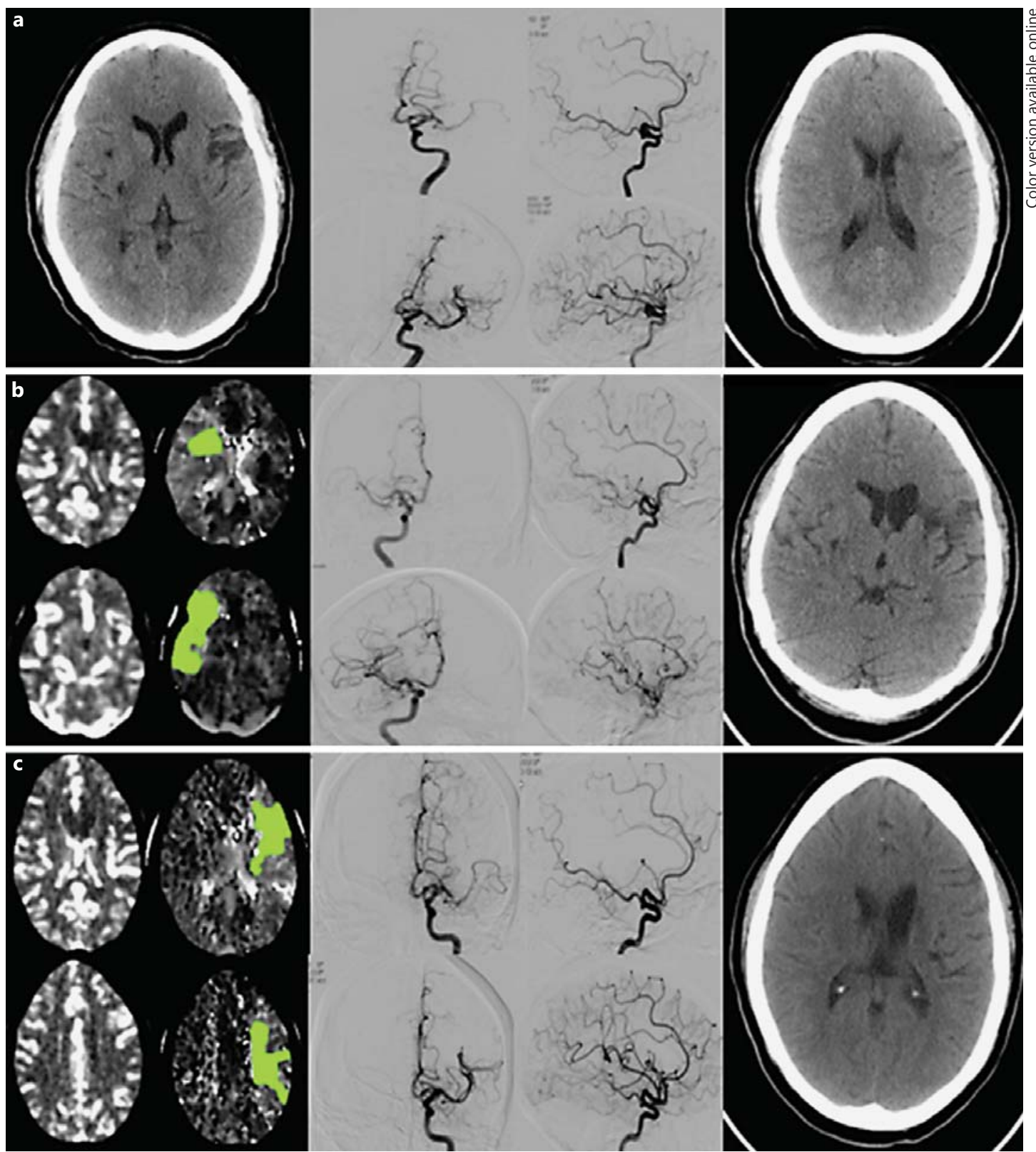

Fig. 1. A 32-year-old women with triple thrombectomy (table 1, case No. 5). a Patient presenting with acute left hemiplegia and aphasia: pre-procedure noncontrast computed tomography (CT) (left), pre- and postprocedure angiography (middle) and follow-up CT (right). b Twenty months later, new-onset right leg weakness: pre-procedure CT perfusion (left), pre- and post-procedure angiography (middle) and follow-up CT (right). c Six weeks after the second intervention, new-onset left side weakness and aphasia: pre-procedure CT perfusion (left), pre- and post-procedure angiography (middle) and follow-up CT (right).

Considering that nearly 1 in 4 patients will have a recurrent stroke within the first 5 years following an AIS [2], and that LVOS accounts for nearly 50\% of all strokes [14], the need for further validation of repeat thrombectomy becomes apparent. Although repeated systemic thrombolysis has been reported to be safe and efficient for patients presenting with a recurrent AIS [3-6], its use carries the limitations inherent to tissue plasminogen activator ( $t-P A)$ including bleeding risk mostly if the recurrence is relatively early on [15]. Intravenous 
Bouslama et al.: Repeated Mechanical Thrombectomy in Recurrent Large Vessel Occlusion Acute Ischemic Stroke

Table 2. Comparison between patients with repeated treatment and patients with single procedure

\begin{tabular}{|c|c|c|c|}
\hline & $\begin{array}{l}\text { Repeated thrombectomy } \\
\text { (last procedure) }(n=15)\end{array}$ & $\begin{array}{l}\text { Single thrombectomy } \\
(\mathrm{n}=557)\end{array}$ & $\mathrm{p}$ value \\
\hline Age, years & $63.4 \pm 15.01$ & $65.4 \pm 14.61$ & 0.61 \\
\hline Gender, male & $6(40 \%)$ & 303 (54.4\%) & 0.3 \\
\hline Hypertension & $15(100 \%)$ & $408(73.4 \%)$ & 0.01 \\
\hline Dyslipidemia & $5(33.3 \%)$ & $230(41.4 \%)$ & 0.6 \\
\hline Atrial fibrillation & $8(53.3 \%)$ & 199 (35.9\%) & 0.18 \\
\hline Diabetes & $2(13.3 \%)$ & $129(23.2 \%)$ & 0.53 \\
\hline Smoking & $3(20 \%)$ & 107 (19.3\%) & 1.00 \\
\hline Anticoagulation & $8(53.3 \%)$ & 75 (16.3\%) & 0.001 \\
\hline Glucose & $122 \pm 28$ & $136 \pm 55$ & 0.08 \\
\hline INR & $1.49 \pm 0.79$ & $1.11 \pm 0.36$ & 0.11 \\
\hline SBP & $138 \pm 23$ & $148 \pm 32$ & 0.12 \\
\hline Baseline NIHSS & $21.21 \pm 7.27$ & $17.9 \pm 6.4$ & 0.11 \\
\hline ASPECTS & $8.36 \pm 1.55$ & $8 \pm 1.7$ & 0.35 \\
\hline Stroke etiology & & & 0.3 \\
\hline Cardioembolic & $10(66.7 \%)$ & 277 (51.3\%) & \\
\hline LVA & $1(6.7 \%)$ & $100(18.5 \%)$ & \\
\hline ICAD & $2(13.3 \%)$ & $42(7.8 \%)$ & \\
\hline Other & $2(13.3 \%)$ & $32(5.7 \%)$ & \\
\hline Undetermined & $0(0 \%)$ & $89(16.5 \%)$ & \\
\hline \multicolumn{4}{|l|}{ Stroke location } \\
\hline ACA & $0(0 \%)$ & $16(2.9 \%)$ & 1.00 \\
\hline MCA-M1 & $9(60 \%)$ & $244(43.8 \%)$ & 0.29 \\
\hline MCA-M2/M3 & $0(0 \%)$ & 98 (17.6\%) & 0.08 \\
\hline ICA terminus & $2(13.3 \%)$ & 77 (13.8\%) & 0.32 \\
\hline Extracranial ICA & $0(0 \%)$ & $15(2.7 \%)$ & 1.00 \\
\hline Vertebrobasilar & $1(6.7 \%)$ & $53(9.5 \%)$ & 1.00 \\
\hline Tandem & $3(20 \%)$ & $70(12.6 \%)$ & 0.42 \\
\hline \multicolumn{4}{|l|}{ Procedure } \\
\hline LKN to puncture & $486 \pm 1,131$ & $325 \pm 297$ & 0.60 \\
\hline Stentriever & $13(86.7 \%)$ & $470(84.4 \%)$ & 1.00 \\
\hline \multicolumn{4}{|l|}{ Reperfusion } \\
\hline mTICI 2b--3 & $15(100 \%)$ & $484(87.2 \%)$ & 0.23 \\
\hline mTICI 3 & $6(40 \%)$ & $263(47.5 \%)$ & 0.61 \\
\hline Parenchymal hemorrhage & $2(13.3 \%)$ & $49(8.8 \%)$ & 0.63 \\
\hline mRS $0--2$ at 3 months & $9(60 \%)$ & $236(46.5 \%)$ & 0.43 \\
\hline Mortality 90 days & $3(20 \%)$ & $114(22.5 \%)$ & 1.00 \\
\hline
\end{tabular}

$\mathrm{ACA}=$ Anterior cerebral artery; ICA = internal carotid artery; ICAD = intracranial atherosclerotic disease; INR = international normalized ratio; LKN = last known normal; LVA = large vessel atherosclerosis; MCA = middle cerebral artery; SBP = systolic blood pressure.

t-PA re-administration has also been associated with severe immune reactions $[16,17]$. The majority of our patients had a cardioembolic etiology, which has been described in the literature to be more prone to stroke recurrence with a risk varying between 1 and $22 \%$ and a mean time between the 2 events of 12 days [18]. Contrarily to extracranial atherosclerosis, the rate of intracranial atherosclerotic disease was higher than usually reported $[19,20]$, and was probably due to a selection bias since our database only includes LVOS treated via endovascular therapy.

Mechanical thrombectomy has proven to be superior to systemic thrombolysis alone in recent clinical trials for LVOS [1] and may be considered as a potential option for patients presenting with a recurrent stroke. Data regarding the repeated use of intra-arterial treatment 
(IAT) is limited to a total of 5 cases [4, 7-9]. Four of these patients underwent 2 IAT and one was treated thrice. Mechanical thrombectomy with stentrievers was performed in 5 out of the 11 procedures and intra-arterial thrombolytic injection was the main treatment in 3 procedures. The mean age was $60.8 \pm 12$, three were male and the median time between procedures was $6(2.5-165)$ days. All patients were successfully reperfused and there were no procedure-related complications following the repeated treatment with good outcomes achieved in $40 \%$ of the cases. It is also noteworthy that $4 / 5$ patients in the above-mentioned case reports had a contraindication to intravenous t-PA at the time of the repeated intervention. Our study confirmed these preliminary findings and showed that when compared with patients that had only one intervention, properly selected RT patients (whether presenting with an early re-occlusion or with completely separate ischemic event) behaved similarly and had high rates of successful reperfusion, acceptable incidence of hemorrhagic complication rates, and high rates of good clinical outcomes.

There are important limitations to our study, mostly inherent to the retrospective design and relatively small sample size. However, to our knowledge, this is the first systematic analysis and represents the largest report on this topic. In our study, only $2 \%$ of the IAT treated patients had recurrent LVOS amenable to a second endovascular treatment. This rate is lower than expected and likely underestimates the true frequency of the problem. There are many factors that might have led to a potential underestimation including the fact that patients with recurrent strokes who were severely disabled after their initial stroke were presumably deemed noncandidates for RT. Moreover, it is likely that there were recurrent strokes that were in territories too distal for IAT, responded to intravenous t-PA, already had large/complete infarcts upon presentation, or were seen in other stroke centers.

\section{Conclusions}

In properly selected patients suffering from recurrent LVOS, repeated mechanical thrombectomy appears to be feasible and safe. A previous thrombectomy should not discourage aggressive treatment as these patients may achieve similar rates of good clinical outcomes as those who undergo single thrombectomy.

\section{Disclosure Statement}

M.B., D.C.H., L.C.R., J.A.G., M.R.F.: no disclosures. R.G.N.: Stryker Neurovacular (Trevo-2 Trial PI, DAWN Trial PI), Covidien (SWIFT and SWIFT-PRIME Steering Committee, STAR Trial Core Lab), and Penumbra (3-D Separator Trial Executive Committee). This research received no specific grant from any funding agency.

\section{References}

1 Goyal M, Menon BK, van Zwam WH, Dippel DW, Mitchell PJ, Demchuk AM, Davalos A, Majoie CB, van der Lugt A, de Miquel MA, Donnan GA, Roos YB, Bonafe A, Jahan R, Diener HC, van den Berg LA, Levy EI, Berkhemer OA, Pereira VM, Rempel J, Millan M, Davis SM, Roy D, Thornton J, Roman LS, Ribo M, Beumer D, Stouch B, Brown S, Campbell BC, van Oostenbrugge RJ, Saver JL, Hill MD, Jovin TG; HERMES collaborators: Endovascular thrombectomy after large-vessel ischaemic stroke: a meta-analysis of individual patient data from five randomised trials. Lancet 2016;387:1723-1731.

2 Mohan KM, Wolfe CD, Rudd AG, Heuschmann PU, Kolominsky-Rabas PL, Grieve AP: Risk and cumulative risk of stroke recurrence: a systematic review and meta-analysis. Stroke 2011;42:1489-1494.

3 Sauer R, Huttner HB, Breuer L, Engelhorn T, Schellinger PD, Schwab S, Kohrmann M: Repeated thrombolysis for chronologically separated ischemic strokes: a case series. Stroke 2010;41:1829-1832. 
4 Yoo HS, Kim YD, Lee HS, Song D, Song TJ, Kim BM, Kim DJ, Kim DI, Heo JH, Nam HS: Repeated thrombolytic therapy in patients with recurrent acute ischemic stroke. J Stroke 2013;15:182-188.

5 Qureshi AI, Malik AA, Freese M, Thompson MJ, Khan AA, Suri MF: Readministration of intravenous alteplase in acute ischemic stroke patients: case series and systematic review. Am J Emerg Med 2015;33:307.e1-4.

6 Cappellari M, Tomelleri G, Carletti M, Bovi P, Moretto G: Intravenous thrombolysis on early recurrent cardioembolic stroke: 'Dr Jekyll' or 'Mr Hyde'? Blood Coagul Fibrinolysis 2012;23:78-81.

7 Lee Y, Yi H, Kim BM, Kim DJ, Kim SH, Nam HS, Heo JH, Kim YD: Recurrent cardioembolic stroke treated successfully with repeated mechanical thrombectomy within the acute index stroke period. J Clin Neurol 2015;11: 275-278.

8 Laible M, Mohlenbruch M, Hacke W, Bendszus M, Ringleb PA, Rizos T: Repeated intra-arterial thrombectomy within 72 hours in a patient with a clear contraindication for intravenous thrombolysis. Case Rep Vasc Med 2015;2015:872817.

9 Suzuki S, Kidwell CS, Starkman S, Saver JL, Duckwiler G, Vinuela F, Ovbiagele B: Use of multimodal MRI and novel endovascular therapies in a patient ineligible for intravenous tissue plasminogen activator. Stroke 2005;36:e77-e79.

10 Zaidat 00, Yoo AJ, Khatri P, Tomsick TA, von Kummer R, Saver JL, Marks MP, Prabhakaran S, Kallmes DF, Fitzsimmons BF, Mocco J, Wardlaw JM, Barnwell SL, Jovin TG, Linfante I, Siddiqui AH, Alexander MJ, Hirsch JA, Wintermark M, Albers G, Woo HH, Heck DV, Lev M, Aviv R, Hacke W, Warach S, Broderick J, Derdeyn CP, Furlan A, Nogueira RG, Yavagal DR, Goyal M, Demchuk AM, Bendszus M, Liebeskind DS; Cerebral Angiographic Revascularization Grading (CARG) Collaborators; STIR Revascularization working group; STIR Thrombolysis in Cerebral Infarction (TICI) Task Force: Recommendations on angiographic revascularization grading standards for acute ischemic stroke: a consensus statement. Stroke 2013;44:2650-2663.

11 Hacke W, Kaste M, Fieschi C, Toni D, Lesaffre E, von Kummer R, Boysen G, Bluhmki E, Hoxter G, Mahagne MH, et al: Intravenous thrombolysis with recombinant tissue plasminogen activator for acute hemispheric stroke. The European Cooperative Acute Stroke Study (ECASS). JAMA 1995;274:1017-1025.

12 Sulter G, Steen C, De Keyser J: Use of the Barthel index and modified Rankin scale in acute stroke trials. Stroke 1999;30:1538-1541.

13 Pexman JH, Barber PA, Hill MD, Sevick RJ, Demchuk AM, Hudon ME, Hu WY, Buchan AM: Use of the Alberta Stroke Program Early CT Score (ASPECTS) for assessing CT scans in patients with acute stroke. AJNR Am J Neuroradiol 2001;22:1534-1542.

14 Smith WS, Lev MH, English JD, Camargo EC, Chou M, Johnston SC, Gonzalez G, Schaefer PW, Dillon WP, Koroshetz WJ, Furie KL: Significance of large vessel intracranial occlusion causing acute ischemic stroke and TIA. Stroke 2009;40:3834-3840.

15 Fugate JE, Rabinstein AA: Absolute and relative contraindications to IV rt-PA for acute ischemic stroke. Neurohospitalist 2015;5:110-121.

16 Pechlaner C, Knapp E, Wiedermann CJ: Hypersensitivity reactions associated with recombinant tissue-type plasminogen activator and urokinase. Blood Coagul Fibrinolysis 2001;12:491-494.

17 Rudolf J, Grond M, Prince WS, Schmulling S, Heiss WD: Evidence of anaphylaxy after alteplase infusion. Stroke 1999;30:1142-1143.

18 Arboix A, Alio J: Cardioembolic stroke: clinical features, specific cardiac disorders and prognosis. Curr Cardiol Rev 2010;6:150-161.

19 Sacco RL, Kargman DE, Gu Q, Zamanillo MC: Race-ethnicity and determinants of intracranial atherosclerotic cerebral infarction. The Northern Manhattan Stroke Study. Stroke 1995;26:14-20.

20 Arenillas JF, Molina CA, Chacon P, Rovira A, Montaner J, Coscojuela P, Sanchez E, Quintana M, Alvarez-Sabin J: High lipoprotein (a), diabetes, and the extent of symptomatic intracranial atherosclerosis. Neurology 2004; 63:27-32. 